\title{
Implementing a Clinical Pathway for Paediatric Mental Health Care in the Emergency Department
}

\author{
Erin M. McCabe \\ University of Alberta \\ Alberta Health Services \\ Kathy GermAnn \\ Lacombe County, Alberta \\ Beverly Lent, and Laurene Black \\ Alberta Health Services \\ Kathryn E. R. Graham \\ Alberta Innovates \\ Douglas P. Gross \\ University of Alberta \\ Maxi Miciak \\ Alberta Innovates, University of Alberta
}

Teresa Katherine Lightbody, Christine Mummery, and Angela Coloumbe

Erin M. McCabe, Department of Rehabilitation Science, University of Alberta; Teresa Katherine Lightbody, Children, Youth \& Families, Addiction and Mental Health, Alberta Health Services - Edmonton Zone; Christine Mummery, Children, Youth \& Families, Addiction and Mental Health, Alberta Health Services - Edmonton Zone; Angela Coloumbe, Children, Youth \& Families, Addiction and Mental Health, Alberta Health Services - Edmonton Zone; Kathy GermAnn, Independent researcher and writer; Beverly Lent, Children, Youth \& Families, Addiction and Mental Health, Alberta Health Services - Edmonton Zone; Laurene Black, Children, Youth \& Families, Addiction and Mental Health, Alberta Health Services - Edmonton Zone; Kathryn Graham, Performance Management and Evaluation, Alberta Innovates; Douglas P. Gross, Department of Physical Therapy \& Rehabilitation Research Centre, University of Alberta; Maxi Miciak, Performance Management and Evaluation, Alberta Innovates \& Faculty of Rehabilitation Medicine, University of Alberta.

Maxi Miciak is now with Maxi Miciak Consulting and the Faculty of Rehabilitation Medicine, University of Alberta. Laurene Black has retired from practice.

This project was financially supported by Alberta Innovates. The authors wish to thank the staff of Alberta Health Services' Stollery Children's Hospital Emergency Department, Mental Health team and their community partners in Edmonton for their contributions to this project. This research was conducted from April 2014 to October 2017. 


\begin{abstract}
Practitioners in emergency departments across Canada are challenged with providing quality mental health $(\mathrm{MH})$ care for children and youth despite increased demand for services. Coordinated service strategies, such as clinical pathways, are needed to effectively manage paediatric MH disorders. Practitioners in a children's hospital emergency department implemented a pathway to improve the care of children and youth with MH conditions. This paper describes an external evaluation of practitioner and stakeholder experiences of the initiative as well as the implementation process, then explores current state and lessons learned. The paper provides a unique contribution to the practitioner-led implementation literature.
\end{abstract}

Keywords: emergency service; mental health; paediatric; care pathway; implementation

\title{
RÉSUMÉ
}

Les praticiens des services d'urgence à travers le Canada doivent relever le défi de fournir des soins de santé mentale (SM) de qualité aux enfants et aux jeunes, et ce, malgré la hausse de la demande pour ces services. La mise en œuvre de stratégies de services coordonnés, tels des parcours cliniques, est nécessaire pour gérer efficacement les problèmes de SM chez les enfants. Les praticiens d'un service d'urgence d'un hôpital pour enfants ont mis sur pied un parcours clinique pour améliorer les soins rendus aux enfants et aux jeunes souffrant de problèmes de SM. Le présent article présente une évaluation externe des expériences des praticiens et des parties prenantes de l'initiative ainsi que le processus de sa mise en œuvre, puis brosse un tableau de la situation actuelle et des enseignements tirés à ce jour. L'article apporte une contribution unique à la littérature portant sur la mise en place d'initiatives dirigées par des praticiens.

Mots clés : services d'urgence ; santé mentale ; pédiatrie ; parcours de soins ; mise en œuvre

Globally, mental health $(\mathrm{MH})$ disorders are a major disease burden for children, youth, and their families. Between 10 to $20 \%$ of Canadian children and youth may be affected by a MH disorder (Canadian Institute for Health Information, 2017). Emergency Departments (EDs) are a common point of access for families and patients seeking MH services. However, EDs are designed to serve patients with a broad spectrum of health conditions, some of which may be life threatening. Care provided in EDs focuses on medical stabilization and risk assessment, which may not be entirely suitable to address the complex nature of the MH concerns of children and youth.

Between 2007 and 2014, there was a 45\% increase in the number of 5- to 24-year-olds presenting to Canadian EDs for MH disorders (Canadian Institute for Health Information, 2015). During the same time period, the prevalence of paediatric $\mathrm{MH}$ disorders and visits to EDs for other concerns remained stable (Canadian Institute for Health Information, 2017; Mapelli, Black, \& Doan, 2015). Much of the increase is attributed to a disproportionate increase in ED visits for non-emergent MH concerns (Mapelli et al., 2015). Additionally, roughly one-third of paediatric MH presentations to the ED are patients returning to the ED,

Correspondence concerning this article should be addressed to Douglas P. Gross, 2-50 Corbett Hall, University of Alberta, Edmonton, AB, T6G 2G4. Email: dgross@ualberta.ca 
which suggests that the needs of children and youth with $\mathrm{MH}$ concerns are not being met in the community (Mapelli et al., 2015; Newton et al., 2010).

Numerous mechanisms have been proposed which may explain this breakdown in the continuity of care for children and youth with MH concerns. There may be a failure to provide adequate assessment, treatment, and follow-up care within the ED; families and primary care providers may not be aware of the existing MH programs within the community; or there may be limited capacity in the community MH services offered (Mapelli et al., 2015; Newton et al., 2010; Newton, Rosychuk, Niu, Radomski, \& McGrath, 2016). To address these challenges, coordinated MH service strategies are needed to (1) improve early identification and intervention for paediatric MH concerns; (2) provide more timely access to community MH services; and (3) reduce service gaps for vulnerable children and youth. Clinical pathways are one strategy that promotes timely and appropriate treatment for children and youth with MH concerns.

A clinical pathway is an accessible, "point of care" clinical decision-making tool that aims to improve the quality of care for a well-defined population of patients (De Bleser et al., 2006). Clinical pathways facilitate the communication, coordination of roles, and sequence of the activities of a multidisciplinary care team (De Bleser et al., 2006). The patient populations with high risk, high volume, or high costs associated with providing their care are usually selected to undergo clinical pathway-based treatment (Jones, 2002). Thus, clinical pathways are especially important in complex clinical environments like hospital EDs, where healthcare providers treat diverse medical conditions, and patient populations, often in chaotic circumstances.

Clinical pathways are often developed internally within hospital settings, and may be limited by a lack of rigor in their development and evidence-based practice expertise among the implementing team (Jabbour et al., 2013). An alternative is the expert-developed clinical pathway, designed for use across a number of health regions and based on high-quality research evidence. An example of an expert-developed care pathway is the Emergency Department Clinical Pathway for Children and Youth with Mental Health Conditions (otherwise known as the emergency department mental health clinical pathway, or EDMHCP) developed by the Ontario Provincial Council for Maternal and Child Health (Provincial Council for Maternal and Child Health, 2013). While expert pathways are usually evidence-based and efficient for standardizing care across health jurisdictions, pathways must be tailored for specific settings, which makes them challenging to implement and evaluate (Jabbour et al., 2013). To overcome the challenge of implementing expert-developed care pathways, a carefully planned intervention strategy is required with direct involvement of practitioners and management who work in and are familiar with the local context (Jabbour et al., 2016). Currently, there is a gap in available evidence regarding the best strategies for implementing clinical pathways (Evans-Lacko, Jarrett, McCrone, \& Thornicroft, 2010; Simmons \& Kotagal, 2008).

\section{Purpose and Overview}

This paper describes the practitioner-led implementation of an expert-developed clinical pathway within the local context of the Stollery Children's Hospital ED in Edmonton, Canada. This study adds to the understanding of why and under what circumstances clinical pathways are successfully implemented. First, the Sources of Information used in this paper are described. This includes a detailed description of an external evaluation of the project. Next, Background contains a description of the contextual factors that led 
to the implementation of the clinical pathway in Edmonton. In Implementation Process, the development of the implementation approach and processes are described. Finally, progress towards the project objectives and the lessons learned are explored in Current State and Lessons Learned.

\section{Sources of Information}

This project was reviewed by the Health Research Ethics Board at the University of Alberta and was determined to be a quality improvement study outside the mandate of the Research Ethics Board per Article 2.5 of the Tri-Council Policy Statement: Ethical Conduct for Research Involving Humans. The sources of information in this paper include an external evaluator report, data used for program monitoring and evaluation in the Stollery ED, and publicly available health service utilization data.

External evaluation report. An external evaluator (KGA) used qualitative and quantitative research methods to capture stakeholders' perspectives of the clinical pathway implementation process. Data were collected through four methods: interviews with key informants, a document review, informal conversations between the evaluator and participants; and quantitative data sources (monitoring data, described below).

Data collection and analysis were iterative. Interview data were collected via 18 group and individual interviews conducted in the summer and fall of 2015. A total of 23 key informants participated, representing the following divisions: management from Child and Adolescent Mental Health Services and the Stollery ED; front line staff from the Stollery ED (ED nursing staff and MH team members); child psychiatrists; and others involved in implementing the ED Pathway. No patients were involved in these interviews. The interviews were semi-structured and lasted between 20 and 90 minutes. There were three interview guides, one for front line staff, one for management, and one for physicians. Questions in the interview guide focused on participants' experiences and lessons learned implementing the ED care pathway. The interviews were audio-recorded and transcribed. Brief field notes were written after each interview, which included the evaluator's observations and questions for further exploration. The interview guide was revised to reflect the iterative process. All participants consented to the audio recording and analysis.

Thematic analysis, a method for identifying, analyzing and reporting patterns (themes) within qualitative data (Braun \& Clarke, 2006), was used to analyze the interview data. Interview data were generated and analyzed concurrently, beginning after the first interview. The evaluator coded and analyzed the interview transcripts and field notes using an iterative process to identify and refine emerging themes and sub-themes within the interview data. These themes were summarized and shared with participants. However, challenges related to limited time and resources, and participant access, precluded an opportunity to obtain feedback from all participants. In addition, not all participants approached for feedback responded to the request. A meeting was held with the available participants and the evaluator to discuss the findings, which generated additional data. The feedback and the additional information were integrated into the analysis.

The evaluator reviewed documents and the performance monitoring data, and extracted information related to the implementation process and the context of children and youth MH services in Edmonton. Informal conversations with practitioners and managers in the ED provided an opportunity for the evaluator to ask questions and seek clarification on aspects of the implementation process. A final written report 
was prepared, which integrates the various data sources to describe the clinical pathway implementation, stakeholder experiences, and lessons learned during the process.

The aim of the external evaluation was to capture the experiences of practitioners and stakeholders involved in this practitioner-led initiative and describe lessons learned to improve the care of children and youth with MH conditions. Therefore, the scope of the evaluation was limited and did not specifically assess effectiveness of the implementation process. However, parts of the evaluator's report describing perspectives and experiences with implementing the clinical pathway were used in this article.

Performance monitoring data. Monitoring is the systematic process of collecting, analyzing and using information to track a program's progress toward reaching its objectives and to guide management decisions (United Nations Entity for Gender Equality and the Empowerment of Women, 2013). We use information collected and analyzed for the purpose of monitoring the EDMHCP implementation in the Stollery ED in this paper. The data describe characteristics of children and youth mental health presentations to the Stollery Hospital ED between April 2014 and April 2017. Data collection and analysis are described below in Phase 3: Planning the implementation strategy.

Interactive Health Data Application. Health service utilization data for the Edmonton region between January 2001-June 2017 were extracted from the Interactive Health Data Application, from the Alberta Health, Surveillance \& Assessment Branch (Alberta Health, 2017).

\section{CONTEXT}

In Edmonton, Canada, the Children, Youth \& Families, Addiction \& Mental Health program (CYFAMH) is responsible for delivering paediatric MH services. CYFAMH is part of Alberta Health Services, the single health authority for the Canadian province of Alberta, which delivers medical care on behalf of the Government of Alberta's Ministry of Health. The Edmonton Zone is one of five geographic areas within Alberta Health Services, serving approximately 1.3 million people in Edmonton and surrounding area. Rates of paediatric ED presentations for MH concerns have been increasing in Alberta. In 2002, there were 126.9 ED visits per 100,000 for an anxiety or stress disorder. This rose to 169.0 per 100,000 in 2010 (Newton et al., 2016). ED visit rates for children and youth for any mental health disorder grew by 41\% from 2004 to 2016 (Alberta Health, 2017). Specific to Edmonton, the number of 15- to 19-year-olds presenting to the ED for anxiety disorders has been steadily rising since 2004 (Health Trends Alberta, 2017).

In February 2014, there was a major change to MH emergency services at the institution level. Child and youth $\mathrm{MH}$ emergency services were relocated from the ED of an adult hospital, to the paediatric ED at the Stollery Children's Hospital. This included integrating a team of mental health nurses (herein, MH team) into the Stollery ED. Coinciding with the move, there was an exponential increase in the number of $\mathrm{MH}$ presentations at the Stollery ED. This created a number of challenges for the staff of the ED at the Stollery (herein, ED staff) identified in the interviews with the external evaluator.

Overall, the relocation of $\mathrm{MH}$ services was described as a taxing experience for patients, the ED staff and physicians, and the MH team. The ED staff members reported feeling overwhelmed with the volume of patients with $\mathrm{MH}$ concerns, and underprepared to manage the complex and challenging behaviours that some of these patients presented. Similarly, newly integrated MH clinicians described feeling uncomfortable 
in their abilities to treat the medical aspects of emergency MH care, having previously worked with children and youth with $\mathrm{MH}$ emergencies on a consultative basis. Additionally, it was found that families in the general paediatric ED waiting room had expressed safety concerns regarding the aggressive or violent behaviours displayed by some patients waiting for MH services.

Practitioners and managers working in the Stollery ED recognized that a better way of organizing and providing $\mathrm{MH}$ care in the Stollery ED was needed. To address the challenges above, in April 2014 the EDMHCP was implemented in Edmonton, with the intention of improving care for children and youth with $\mathrm{MH}$ emergencies in the region.

\section{IMPLEMENTATION PROCESS}

The implementation of the EDMHCP was carried out by a working group of practitioners and management from the Stollery ED and the CYFAMH program. The process is described in four phases. Phase 1 involved identifying challenges and objectives, which informed phase 2, developing an approach. Following this, implementation strategy planning occurred in phase 3, and finally, the EDMHCP was implemented in phase 4 . These phases are described in the following sections.

\section{Phase 1: Defining Objectives}

The managers from the Stollery ED and CYFAMH program met frequently to discuss challenges. The working group identified the following four key objectives to focus the development of an approach to improve quality of MH services for children and youth in Edmonton:

1. build capacity to care for children and youth presenting to the ED with MH concerns;

2. ensure appropriate and timely follow-up care for children and youth with MH concerns after discharge home from the ED;

3. partner with community organizations to enhance their capacity to support children and youth experiencing $\mathrm{MH}$ concerns in the community; and

4. reduce the number of children and youth presenting to the ED with non-urgent MH concerns.

\section{Phase 2: Developing the Approach}

The working group explored the literature for potential solutions that would be consistent with their objectives. The EDMHCP was chosen because its goals were congruent with the objectives identified by the group, it was evidence-based, and it had an associated implementation toolkit. The working group anticipated that implementing the EDMHCP would support effective and timely follow-up in the community. They also intended that it would build capacity in the community to effectively manage $\mathrm{MH}$ concerns with the aim of reducing the number of visits to the ED for non-emergent MH concerns. Additionally, it was believed that the EDMHCP would enhance the capacity of the ED to provide a consistent, basic level of MH care to paediatric $\mathrm{MH}$ patients. The working group obtained support from AHS senior leadership, and secured funding for a project manager to support the implementation of the EDMHCP. 
Table 1

\section{Description of the Emergency Department Clinical Pathway for Children and Youth with Mental Health} Conditions

\begin{tabular}{|c|c|c|}
\hline Core Component & Elements & Purpose \\
\hline \multirow[t]{5}{*}{ High-level algorithm } & $\begin{array}{l}\text { Emergency department (ED) } \\
\text { triage }\end{array}$ & Entry point for patients, triaged by ED nurse \\
\hline & $\begin{array}{l}\text { Resuscitative/emergency care } \\
\text { required }\end{array}$ & Immediate medical stabilization \\
\hline & Mental health screening & Self-report questionnaires \\
\hline & Clinical assessment & By mental health clinician or ED physician \\
\hline & Disposition & $\begin{array}{l}\text { Admission, referral, discharge home with follow-up } \\
\text { recommendations }\end{array}$ \\
\hline \multirow[t]{3}{*}{ Minimum standards } & $\begin{array}{l}\text { Access to a mental health } \\
\text { clinician }\end{array}$ & Clinicians within the hospital from the community \\
\hline & $\begin{array}{l}\text { Standardized triage screening } \\
\text { tools }\end{array}$ & $\begin{array}{l}\text { "Children's Hospital of Eastern Ontario Caregiver/Youth } \\
\text { Perception Survey," "Ask Suicide Screening Questions," } \\
\text { "Paediatric Symptom Checklist" or "Global Appraisal of } \\
\text { Individual Needs" }\end{array}$ \\
\hline & $\begin{array}{l}\text { Memorandum of } \\
\text { Agreement }\end{array}$ & $\begin{array}{l}\text { Partnership agreement between ED and community MH } \\
\text { service providers }\end{array}$ \\
\hline $\begin{array}{l}\text { Recommended } \\
\text { practices }\end{array}$ & $\begin{array}{l}\text { Pre-printed Physician Order } \\
\text { sets }\end{array}$ & $\begin{array}{l}\text { Point-of-care tool to ensure standardized, evidence-based } \\
\text { management practices. e.g., pre-printed orders for use of } \\
\text { chemical restraints in paediatric patients }\end{array}$ \\
\hline
\end{tabular}

Source: adapted from Implementation Toolkit: Emergency Department Clinical Pathway for Children and Youth with Mental Health Conditions (Provincial Council for Maternal and Child Health, 2013).

Description of the clinical pathway. The EDMHCP was based on an extensive literature review, an environmental scan, and consultation with experts (Provincial Council for Maternal and Child Health, 2013), with the goal of supporting ED staff in providing evidence-based care of children and youth with MH conditions. According to the developers, the EDMHCP defines an evidence-based, standardized approach to risk assessment and discharge decision-making. It aims to improve access to appropriate needs-based $\mathrm{MH}$ care as well as provide a seamless transition of care for children and youth between the ED, community MH services and external community partners, (Provincial Council for Maternal and Child Health, 2013). Table 1 outlines the key components of the EDMHCP. An evaluation of the clinical pathway implementation protocol was conducted in 2015 in Toronto, Canada (Barwick et al., 2015). The evaluation informed improvements and changes to the implantation protocol. A mixed-methods research study to rigorously assess and evaluate the pathway implementation process is currently underway at four sites (Jabbour et al., 2018). The protocol for that study has been published (Jabbour et al., 2016). 


\section{Phase 3: Planning the Implementation Strategy}

The goal of the planning phase was to prepare for implementation of the EDMHCP. This phase had four parts: (1) environmental scan to assess the current state of paediatric MH services in Edmonton; (2) identifying the aspects of the EDMHCP to be targeted for implementation; (3) obtaining leadership support; and (4) planning how to monitor the implementation. Each will be discussed in detail.

Environmental scan. An assessment of the local context of paediatric MH services in Edmonton was conducted through consultation and relationship building with four key stakeholder groups including the Stollery ED staff, Stollery MH team, community-based MH service providers, and external community partners (police, schools, social service agencies). The assessment focused on understanding the barriers to delivering $\mathrm{MH}$ services for children and youth, the responsibilities and priorities of each group, and the referral processes for patient flow in and out of the ED. The assessment process also helped promote awareness of the project and to gain support for the project among community stakeholders.

Identify gaps. The gaps between current practice in Edmonton and EDMHCP pathway recommendations were identified. The practices and processes that required development or change were outlined, and include (a) establishing specific work and care processes for patient flow in the ED; (b) clarifying roles and responsibilities of clinicians working in the Stollery ED; (c) establishing team communication practices and streamlining referral processes to community partners; and (d) staff education regarding patient $\mathrm{MH}$ screening tools.

Leadership support. The need for leadership support was identified. A new managerial role was created whose responsibilities included the MH team in the ED and the internal community MH service providers. The aim was to facilitate implementation of the EDMHCP by giving a manager the authority to introduce and sustain changes in CYFAMH services.

Performance monitoring. As recommended in the EDMHCP implementation toolkit (Provincial Council for Maternal and Child Health, 2013), a plan for monitoring the progress of pathway implementation was made. Considering availability, feasibility of collection, and importance, the working group used three data sources: a data collection form completed by clinical staff in the ED, a shared electronic medical record, and a province-wide ED health utilization database.

The data collection form was developed to gather demographic and clinical information about the paediatric patients visiting the ED for MH concerns. For each patient, data were collected on the source of the referral to the ED, the findings from the clinical assessment, and the plan for follow-up after the patient was discharged from the ED. The team expected these variables to be impacted by the clinical pathway implementation. Clinical data were extracted from a shared electronic medical record system to supplement the data collection form. The team believed that tracking health service utilization and patient flow through the ED was important, given the objectives of the clinical pathway implementation. Therefore, data were also extracted from an existing, province-wide ED health utilization database (the Emergency Department Information System).

A data manager was appointed, who became responsible for collecting, analyzing, and reporting performance monitoring data on a monthly basis. The data manager would conduct exploratory data analysis to examine trends in the number of $\mathrm{MH}$ presentations to the ED over time, analyze ED visit rates by patient 
demographic characteristics (age, sex, patient address/geographic residence, reason for presentation), and health service utilization (discharge plan, source of referral, and ED services accessed, time of day, day of week presented). They may also suggest changes to the types or manner of data being collected. Each month, the data manager would provide an internal report to ED staff, the MH team, and administration. This report would serve to communicate progress and also guide decision-making related to the project objectives.

\section{Phase 4: Implementation}

Based on the local needs and resources available in Edmonton, the working group decided to focus on implementing the following aspects of the EDMHCP: (a) a high-level algorithm that describes key aspects of patient flow through the ED, (b) standardized triage screening tools, and (c) collaborative partnerships with community MH service providers.

Patient flow algorithm. Initially, the working group implemented the original algorithm presented in the EDMHCP. It has been adapted and revised periodically to ensure it is congruent with the context and demands for $\mathrm{MH}$ services (programs, clinicians, service available, and infrastructure) in Edmonton. Modifications were based on feedback from clinicians and community partners, and the performance monitoring data.

Standardized screening tools. The Children's Hospital of Eastern Ontario Caregiver/Youth Perception Survey (CHEO survey) was implemented in the ED. The CHEO survey is a screening tool designed to assess the concerns and expectations of patients presenting in the ED. There are two versions, a self-report survey for patients 12 years of age and older (Youth Perception Survey), and a proxy-report for caregivers of children under 12 years of age (CHEO Caregiver Perception Survey). The other screening tools recommended in the EDMHCP were not implemented.

Partnerships with community. The working group focused on enhancing the availability of community MH services, changing referral patterns of community partners to the Stollery ED, and ensuring that children and youth are connected with appropriate MH services in the community after discharge from the ED.

\section{CURRENT STATE}

Enhancing paediatric MH services in the ED required skillful and adequately resourced efforts to implement and sustain required changes (Evans-Lacko et al., 2010). It is challenging to work with a number of stakeholder groups, with a complexity of priorities and values. As major changes were made to $\mathrm{MH}$ services at the Stollery, the working group found it useful and practical to adopt a monitoring approach focused on ongoing learning and adaptation. This consisted of observing progress towards meeting objectives through collecting and analyzing relevant data, examining trends, and engaging in frequent dialogue with practitioners from the ED staff, MH team, and community partners. By adopting this approach, the working group was successful in implementing and refining a number of aspects of the EDMHCP in the Stollery ED. Children and youth presenting to the Stollery ED with MH concerns are now being seen in a paediatric, family-centred ED with access to paediatric MH expertise. They are triaged, medically stabilized (if required), clinically assessed and, if not admitted, referred to follow-up care. The progress towards each objective is described in the next section. 


\section{Objective 1: Build capacity to care for children and youth presenting to the ED with MH concerns}

The implementation of the EDMHCP was expected to increase the capacity of ED physicians and nurses to care for children and youth with MH concerns. A high-level patient-flow algorithm was developed and is in place in the Stollery ED; it is available as supplementary online material. It was reported that in practice, there is much variability in the degree to which each patient presentation to the ED reliably follows the care map outlined in the algorithm. However, patient flow is improving as ED algorithms are improved.

It was reported that the CHEO surveys are now being used consistently in the Stollery ED. The EDMHCP's recommended minimum standard is around-the-clock access to a consulting MH clinician. This recommendation has been met and exceeded in the Stollery ED, with a MH team situated to deliver care in the Stollery ED for 16 hours per day, access to psychiatry on-call overnight, and MH care aides available around-the-clock.

\section{Objective 2: Ensure appropriate and timely follow-up care for children and youth with MH concerns after discharge from the ED}

A number of changes aimed at increasing the $\mathrm{MH}$ services available in the community have been implemented. A key aspect of the Stollery ED patient care pathway is follow-up care for patients after discharge. The Mobile Response Team (MRT) was an existing community MH service. The working group felt that the MRT was underutilized as a follow-up service for patients after discharge from the ED, because prior to the EDMHCP, only $10 \%$ of patients were referred to the MRT by ED staff. As part of the implementation of the patient flow through the ED algorithm, referral to MRT became a standard part of planning for patient discharge. The proportion of patients with $\mathrm{MH}$ concerns that are referred to this service has since increased (Figure 1). Over the last 12 months of available data, approximately $40 \%$ of children and youth with $\mathrm{MH}$ concerns are now referred to the MRT.

\section{Objective 3: Partner with external community organizations to enhance capacity to support children and youth experiencing MH concerns in the community}

A major focus of the CYFAMH working group was to collaborate with community-based agencies to ensure sufficient capacity in the community to support children and youth in the community prior to crises that lead to an ED visit. External community partners are a significant source of referrals to the ED. When paediatric emergency MH services were relocated to the Stollery ED in 2014, the number of children sent to the Stollery ED by external community partners increased rapidly. A maximum was reached in 2015, when about $40 \%$ of all patient visits to the ED were referrals by external partners (police, schools, social service agencies). CYFAMH managers have made numerous presentations in the community with the aim of increasing awareness of the community MH services that are available as alternatives to the ED. Participants in these sessions have included parents, school personnel, emergency services personnel, and staff of community agencies that support children with $\mathrm{MH}$ diagnoses. The proportion of referrals from external partners has decreased to an average of around $23 \%$ over the last 12 months in which data is available (see Figure 2). 


\section{Figure 1}

The Proportion of Patients with Mental Health Concerns Referred to the Mobile Response Team by ED Clinicians per Month

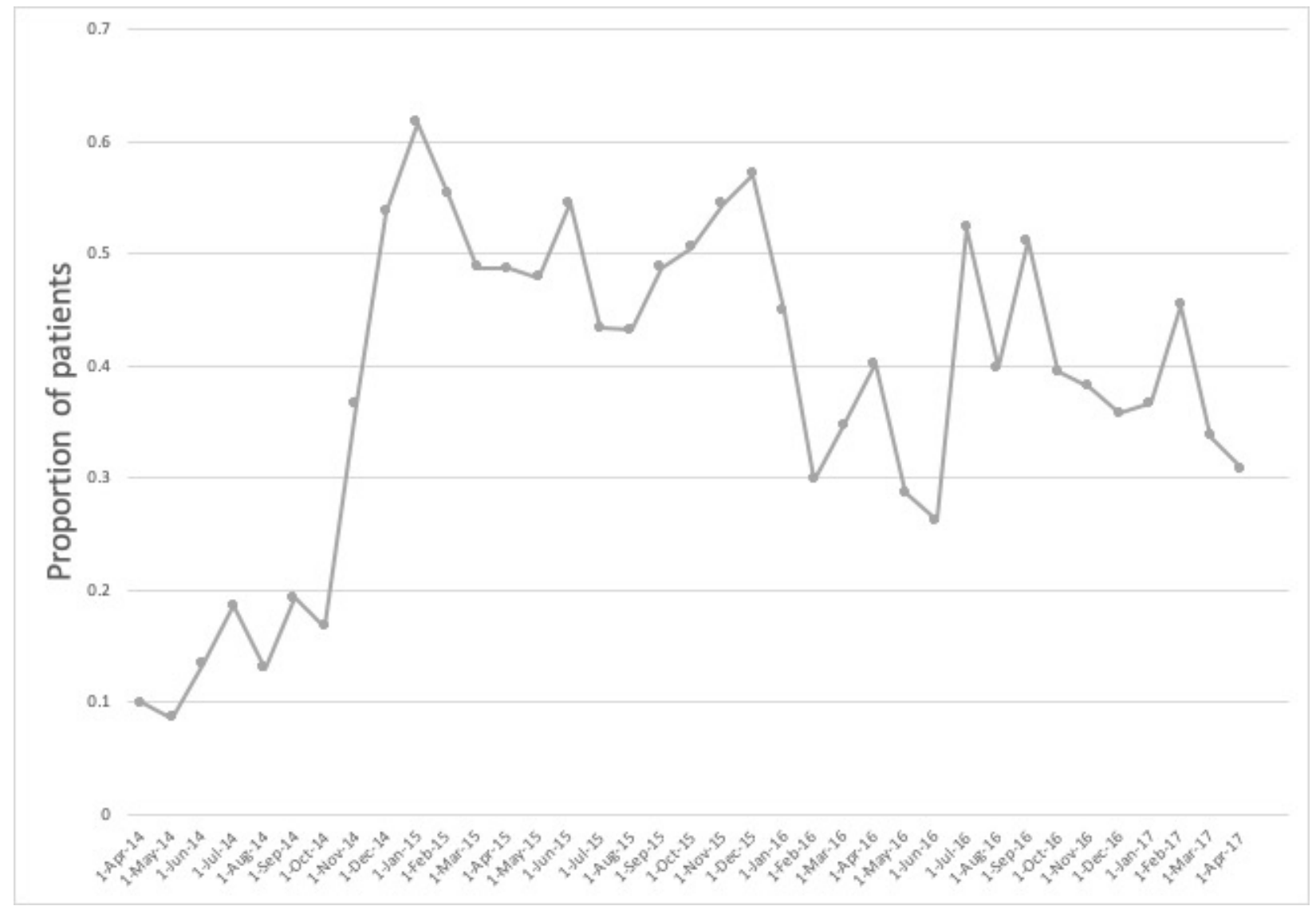

A focused partnership between CYFAMH and Edmonton Police Services was established. A specific aim of the partnership was to reduce the number of children and youth who are apprehended and conveyed to the Stollery ED by a peace officer under Section 12 of the Alberta Mental Health Act. Under Section 12, a person who is believed to be suffering from a mental disorder can be apprehended by a peace officer, and conveyed to a designated facility for examination by a physician, if this is considered to be in the interest of their own safety or the safety of others (Alberta Health Services, 2010). CYFAMH and the police recognized that children and youth who require examination and treatment for a $\mathrm{MH}$ disorder are often better served in community-based services, rather than the ED. Therefore, the police focused on educating their officers about the alternatives to the ED that are available in the community, and training focused on facilitating access to these services by working with caregivers of children and youth in need of MH care. Figure 3 shows a downward trend in the number of children and youth who were apprehended and conveyed to the Stollery ED per month from April 2014 to April 2017. 


\section{Figure 2}

Proportion of Patients Presenting to the Stollery Emergency Department Sent by Community Partner Agencies per Month

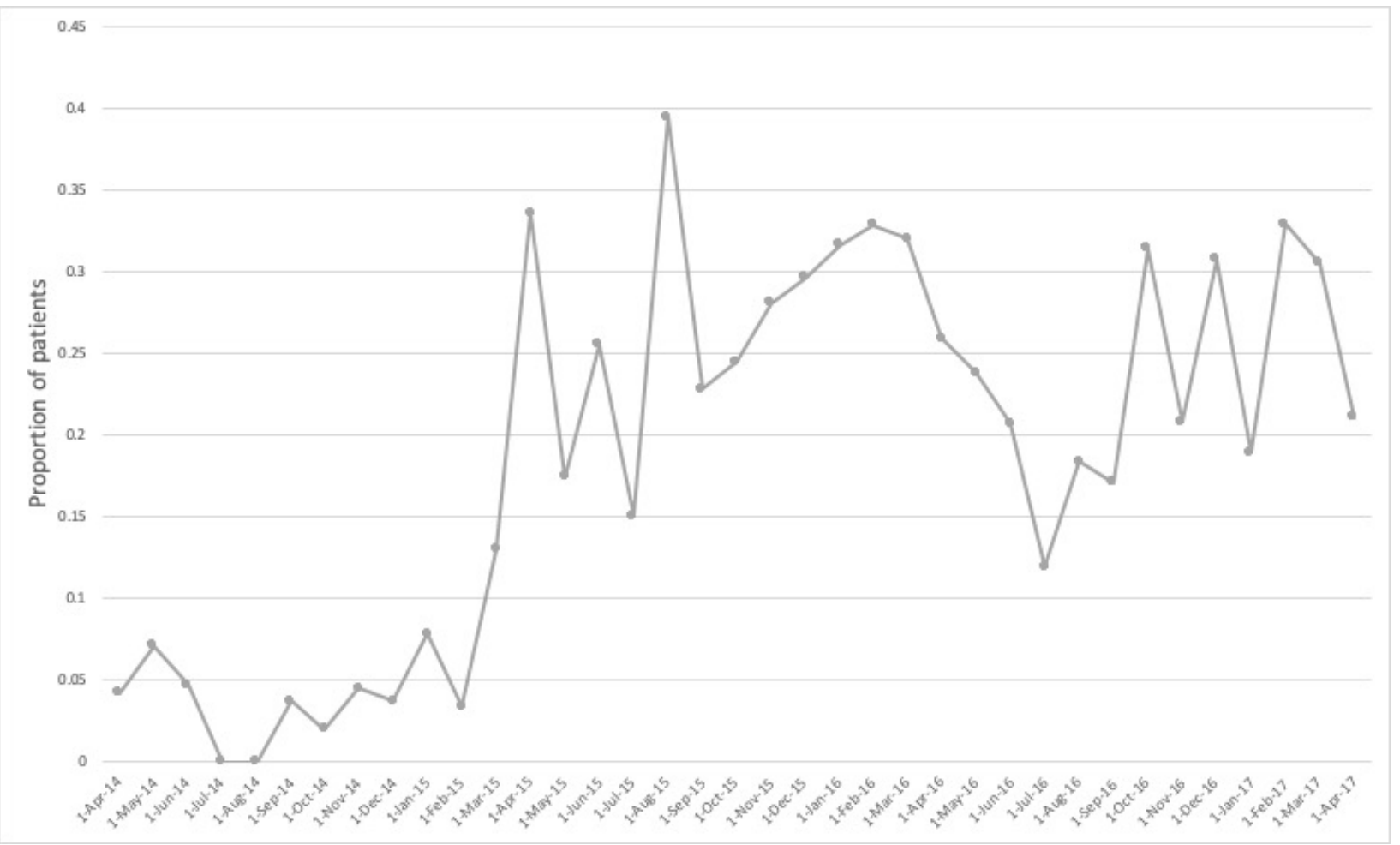




\section{Figure 3}

The Number of Children and Youth Brought to the ED by Peace Officers under Section 12 of the Alberta Mental Health Act per Month

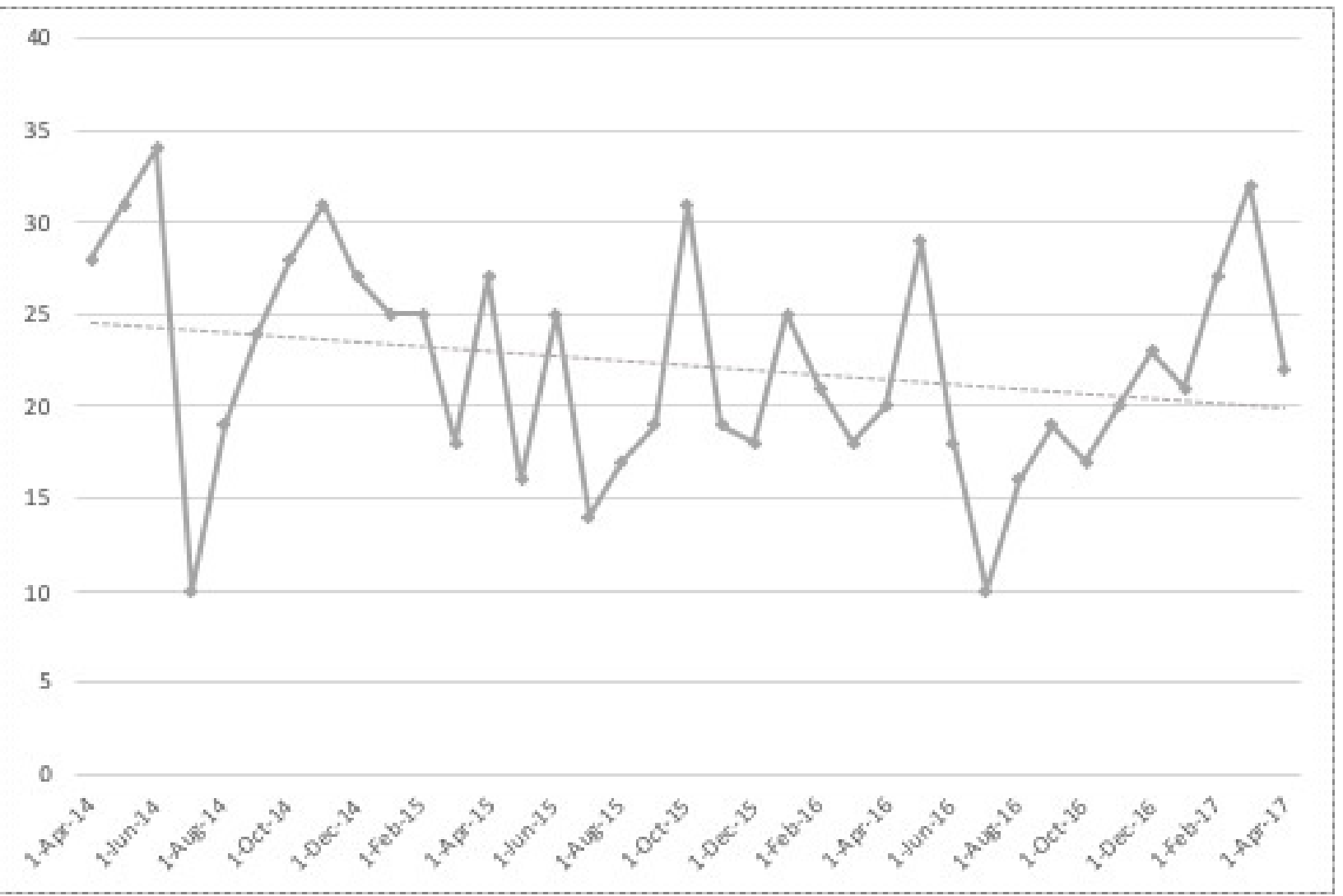

In addition, business partnerships were established between AHS and the Government of Alberta's Department of Disability Services and Department of Children's Services. These agencies will be responsible for urgently responding to youth and families where a home placement (parent home or group home) is breaking down due to extreme behaviours of the child. Usual practice is for these children and youth to be sent to the Stollery ED. These children, youth, and families are likely better served by a home-based intervention. This is expected to decrease ED utilization rates by children, youth, and families in these situations.

Finally, a Statement of Agreement is under development between AHS and each community partner. The intent of a Statement of Agreement is to ensure a focused and active collaboration between the Stollery $\mathrm{ED}$ and the community agency. It aims to promote fair and timely access to children's MH services, prescribe the access and referral pathways for children and youth requesting MH services, establish clear guidelines for the community response to children and youth discharged from the $\mathrm{ED}$, and outlines processes to review ED presentations for ongoing quality improvement. 


\section{Objective 4: Reduce the number of children and youth presenting to the ED with non-urgent MH concerns}

The services at the single-session, walk-in community MH clinic were expanded by five times ( 1 halfday a week to 5 half-days a week). An urgent MH clinic was relocated to the Stollery with the intention of providing children and youth in crisis with more efficient and easier access to psychiatry and MH services on an urgent basis. Despite the changes to the Stollery ED and community MH services, the number of presentations to the Stollery ED has continued to rise. Figure 4 shows the absolute number of visits to the Stollery ED per month from Feb 2014 to May 2017. Figure 5 shows the number of presentations to any ED in Alberta between 2004 and 2015.

\section{DISCUSSION}

The CYFAMH working group has made considerable progress towards implementing the EDMHCP with the aim of improving the quality of $\mathrm{MH}$ services for children and youth in Edmonton. The increasing demand for paediatric MH services and challenge of providing quality care for these patients in the ED is a problem faced by practitioners in EDs across Canada. The working group used an innovative approach in addressing this complex clinical problem. They combined the local knowledge and clinical expertise of practitioners in Edmonton with an evidence-based care pathway, engaged internal and external community

Figure 4

The Absolute Number of Child and Youth Presenting to the Stollery ED per Month from April 2014 to April 2017

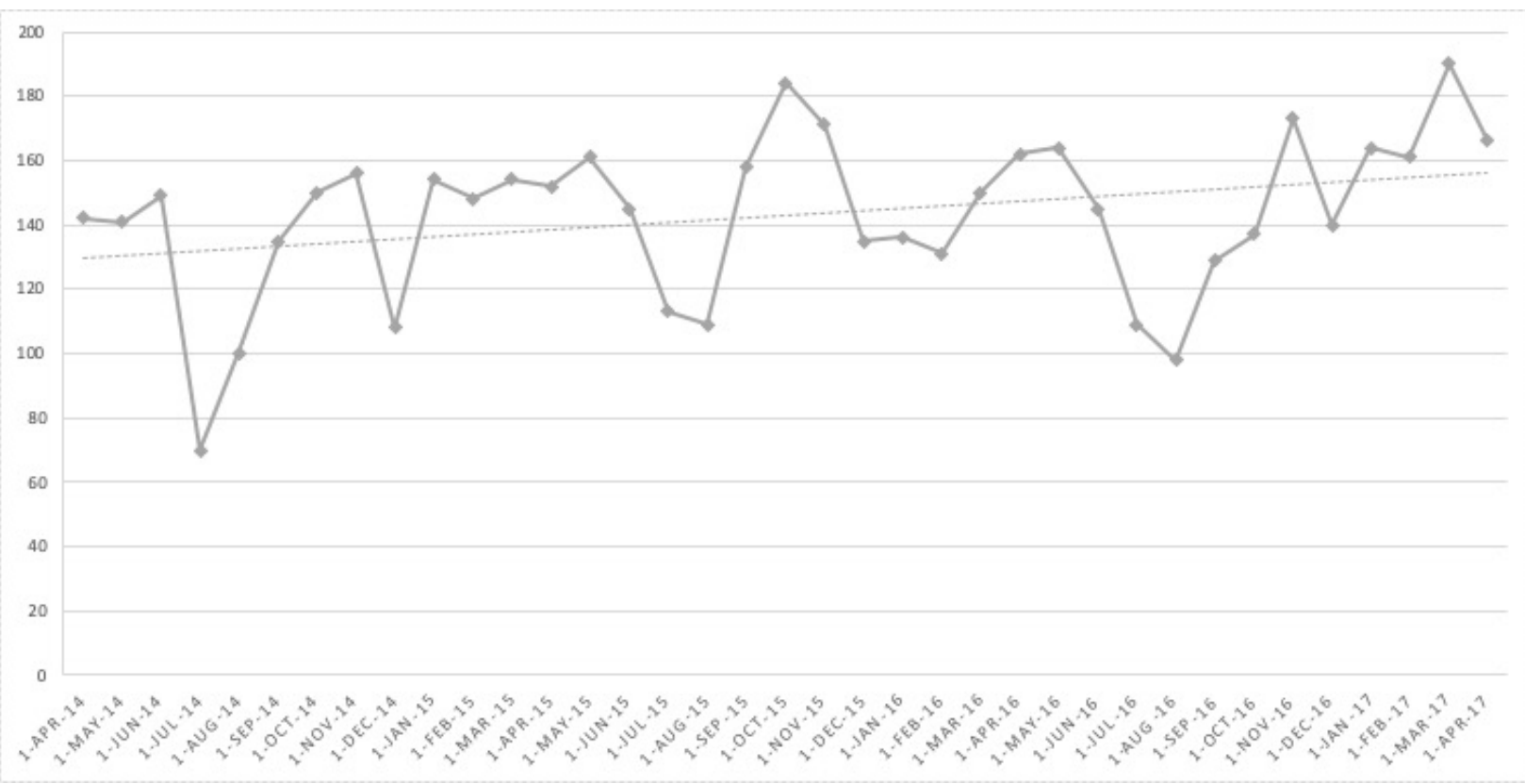




\section{Figure 5}

\section{The Absolute Number of Presentations to an ED in Alberta by Children or Youth with a MH Diagnosis}

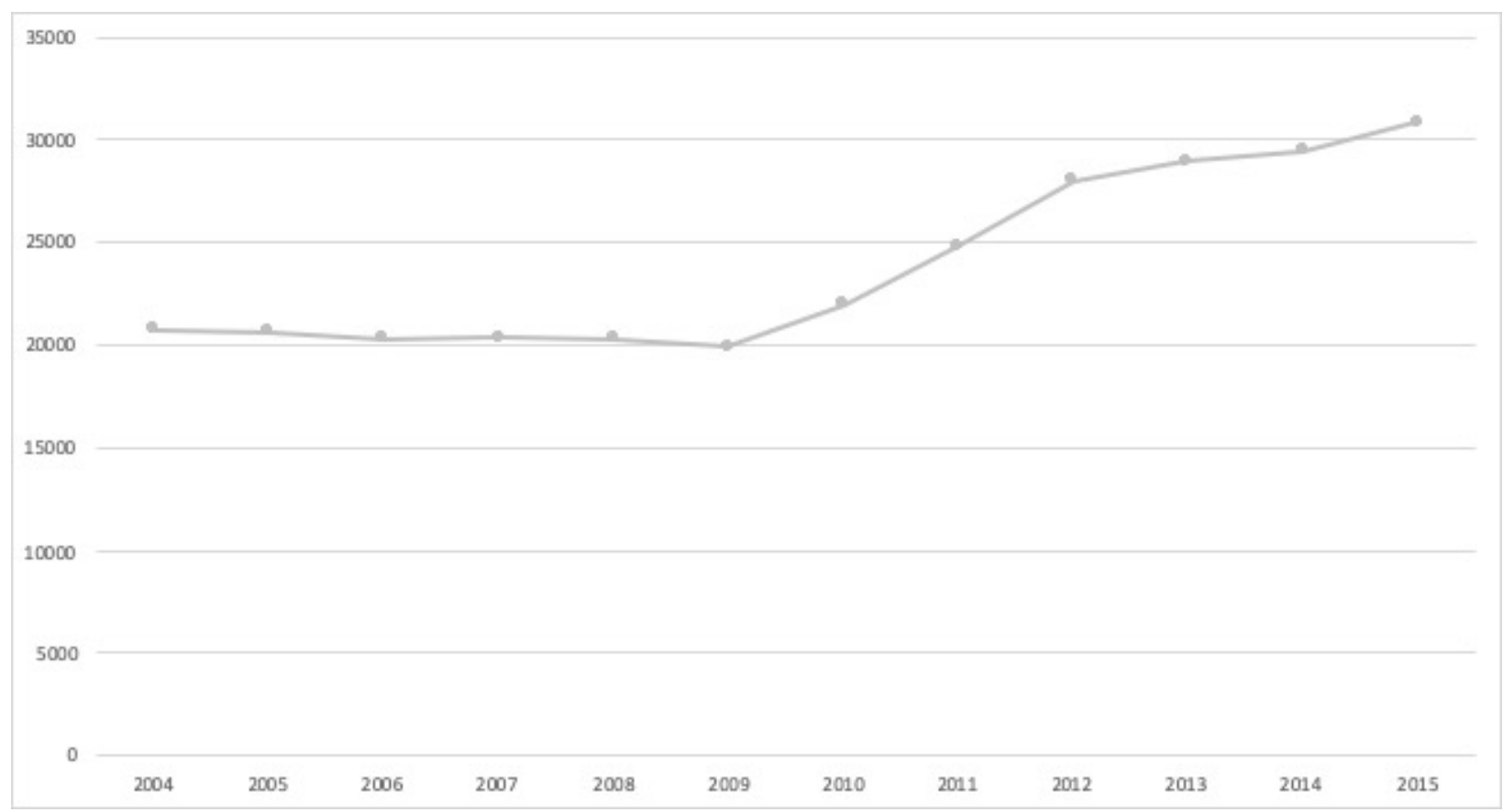

Note. Children and youth defined as 0-19 years of age.

partners, and hired an external evaluator to examine and provide a quality improvement-oriented evaluative report on the implementation process. The practitioners in the Stollery ED took a unique approach to improving MH services, when compared to the findings of Leon et al. (2013), who conducted a survey of the MH services of EDs in paediatric tertiary care centres across Canada. At the time the study was conducted, November 2009 to January 2010, the practices for developing children and youth MH emergency services were highly varied, mostly based on local clinical experience, without a foundation of research evidence, or the involvement of community partners. None of the Canadian EDs reported an external evaluation of their MH services, and few had formal evaluation processes (Leon et al., 2013). An additional distinct feature of the EDMHCP implementation at the Stollery ED was that it was a practitioner-led initiative.

The volume of children and youth presenting to the Stollery ED with MH concerns was greater than initially anticipated after relocating paediatric emergency MH services to the Stollery ED. The extraordinary increase, which has continued since the expansion of MH services at community MH clinics, likely indicates an unmet need for MH services in the community. To meet the growing demand, it may be necessary to continue to expand community MH services in the Edmonton zone. Authors of other publications in the paediatic ED literature have suggested strategies to decrease paediatric ED utilization for non-urgent $\mathrm{MH}$ concerns such as increased awareness of existing community-based $\mathrm{MH}$ services, an intention to refine 
referral processes for families and primary care providers, and to develop early identification and treatment programs for children and youth with MH conditions (Mapelli et al., 2015; Newton et al., 2010; Newton, Rosychuk, Niu, Radomski, \& McGrath, 2016). Gaining a greater understanding of why there is an ongoing increase in paediatric MH utilization rates in Edmonton is worthy of closer investigation.

\section{Lessons Learned}

The practitioners in the Stollery ED encountered challenges during the implementation of the clinical pathway. Unanticipated difficulties observed in the Stollery ED included not all stakeholder groups being fully engaged and invested in the process, and clinicians in the Stollery ED feeling underprepared for the changes in terms of clinical skills training, role clarity, and establishing collaborative working relationships among ED staff, the MH team, and community agencies. An approach focused on greater investment in the preparation and planning stages could have improved the implementation process at the Stollery ED.

A lesson learned from the experience in the Stollery ED is the importance of investing in the preparation and planning stages of clinical pathway implementation. One strategy for planning is to use an implementation protocol, such as the Implementation Plan associated with the EDMHCP. The Implementation Plan provides a structured approach to comprehensively assess the contextual factors specific to successfully implementing the clinical pathway (Provincial Council for Maternal and Child Health, 2013). Following the Implementation Plan would have helped the working group identify potential barriers to implementation during the planning stage, and perhaps anticipate and manage some of the challenges that were encountered more effectively.

The second lesson learned through this initiative relates to assessing the progress and outcomes of this initiative. This grassroots approach to clinical pathway implementation did not include a systematic plan for data collection and evaluation. Consequently, while the CYFAMH working group was successful in implementing aspects of the EDMHCP, it is difficult at present to assess the extent to which these changes have impacted the quality of care for children and youth presenting to the Stollery ED with MH concerns. Establishing an evaluation framework at the outset would have aided with planning the implementation, identifying specific outcomes, and directing choices for methods of data collection and specific analysis tools. Moving forward, it will be important to develop a comprehensive evaluation framework and measurement approach to collecting evidence about implementation. A well-designed evaluation plan would clarify for practitioners what worked and did not work, and under what conditions, in addition to informing decisions about appropriate scale-up, sustainability of, and adaptations to the pathway.

\section{CONCLUSIONS}

This paper describes the processes and impact of the implementation of an expert-developed MH care pathway by practitioners in a paediatric ED. The practitioners and managers from the Stollery ED integrated a $\mathrm{MH}$ team in the ED, worked to improve patient flow, and introduced a validated screening tool. The community MH services in Edmonton were enhanced by the addition of new community MH clinics and expansion of existing MH services. Additionally, a strong foundation of collaboration has been developed 
with external community organizations. On a larger scale, this paper adds to the current body of literature regarding barriers and facilitators influencing the implementation of expert-developed clinical pathways by practitioners, with a specific focus on the growing area of children and youth $\mathrm{MH}$. It offers valuable insight for quality improvement practice in future settings.

\section{REFERENCES}

Alberta Health. (2017). Health Service Utilization - Interactive Health Data Application. Retrieved November 6, 2017, from http://www.ahw.gov.ab.ca/IHDA_Retrieval/selectCategory.do?dataBean.id=122\&command=doSelectSub Category\&cid $=122$

Alberta Health Services. (2010). Guide to the Alberta Mental Health Act and Community Treatment Order Legislation (2010 ed.). Calgary: Alberta Health Services. Retrieved from https://www.albertahealthservices.ca/assets/info/ hp/mha/if-hp-mha-guide.pdf

Barwick, M., Boydell, K., Horning, J., Stasiulis, E., Gladstone, B., \& The Hospital for Sick Children. (2015). Evaluation of Ontario's emergency department clinical pathway for children and youth with mental health conditions. Toronto, ON. https://doi.org/10.13140/RG.2.1.1416.7206

Braun, V., \& Clarke, V. (2006). Using thematic analysis in psychology. Qualitative Research in Psychology, 3(2), 77-101. https://doi.org/10.1191/1478088706qp063oa

Canadian Institute for Health Information. (2015). Care for Children and Youth With Mental Disorders. Retrieved from https://secure.cihi.ca/free products/CIHI CYMH Final for pubs EN web.pdf

Canadian Institute for Health Information. (2017). Child and youth mental health in Canada: Infographic. Retrieved November 6, 2017, from https://www.cihi.ca/en/child-and-youth-mental-health-in-canada-infographic

De Bleser, L., Depreitere, R., De Waele, K., Vanhaecht, K., Vlayen, J., \& Sermeus, W. (2006). Defining pathways. Journal of Nursing Management, 14(July), 553-563. https://doi.org/http://dx.doi.org/10.1111/j.1365-2934.2006.00702. x

Evans-Lacko, S., Jarrett, M., McCrone, P., \& Thornicroft, G. (2010). Facilitators and barriers to implementing clinical care pathways. BMC Health Services Research, 10, 182. https://doi.org/10.1186/1472-6963-10-182

Health Trends Alberta. (2017). Anxiety Disorders among Teens in Alberta.

Jabbour, M., Curran, J., Scott, S. D., Guttman, A., Rotter, T., Ducharme, F. M., ... Johnson, D. W. (2013). Best strategies to implement clinical pathways in an emergency department setting: study protocol for a cluster randomized controlled trial. Implementation Science, 8(1), 55. https://doi.org/10.1186/1748-5908-8-55

Jabbour, M., Hawkins, J., Day, D., Cloutier, P., Polihronis, C., Cappelli, M., ... Gray, C. (2018). An Emergency Department Clinical Pathway for Children and Youth with Mental Health Conditions. Child and Adolescent Psychiatric Clinics of North America, 27(3), 413-425. https://doi.org/10.1016/j.chc.2018.02.005

Jabbour, M., Reid, S., Polihronis, C., Cloutier, P., Gardner, W., Kennedy, A., ... Cappelli, M. (2016). Improving mental health care transitions for children and youth: a protocol to implement and evaluate an emergency department clinical pathway. Implementation Science, 11(90), 1-9. https://doi.org/10.1186/s13012-016-0456-9

Jones, S. (2002). A clinical pathway for pediatric gastroenteritis. Gastroenterology Nursing : The Official Journal of the Society of Gastroenterology Nurses and Associates, 26(1), 7-18. https://doi.org/00001610-200301000-00003 [pii]

Leon, S. L., Cappelli, M., Ali, S., Craig, W., Curran, J., Gokiert, R., ... Newton, A. S. (2013). The current state of mental health services in Canada's paediatric emergency departments. Paediatrics \& Child Health, 18(2), 81-85. https:// doi.org/https://doi.org/10.1093/pch/18.2.81

Mapelli, E., Black, T., \& Doan, Q. (2015). Trends in Pediatric Emergency Department Utilization for Mental HealthRelated Visits. The Journal of Pediatrics, 167(4), 905-910. https://doi.org/10.1016/j.jpeds.2015.07.004

Newton, A. S., Ali, S., Johnson, D. W., Haines, C., Rosychuk, R. J., Keaschuk, R. A., ... Klassen, T. P. (2010). Who comes back? Characteristics and predictors of return to emergency department services for pediatric mental health care. Academic Emergency Medicine, 17(2), 177-186. https://doi.org/10.1111/j.1553-2712.2009.00633.x

Newton, A. S., Rosychuk, R. J., Niu, X., Radomski, A. D., \& McGrath, P. J. (2016). Emergency Department Use and Postvisit Care for Anxiety and Stress Disorders Among Children. Pediatric Emergency Care, 32(10), 658-663. https://doi.org/10.1097/PEC.0000000000000747 
Provincial Council for Maternal and Child Health. (2013). Implementation Toolkit: Emergency Department Clinical Pathway for Children and Youth with Mental Health Conditions.

Simmons, J. M., \& Kotagal, U. R. (2008). Reliable Implementation of Clinical Pathways: What Will It Take -That Is the Question. Journal of Pediatrics, 152(3), 303-304. https://doi.org/10.1016/j.jpeds.2007.12.017

United Nations Entity for Gender Equality and the Empowerment of Women. (2013). Section IV. Monitoring and Evaluation. In Ending Violence Against Women and Girls: Programming Essentials (p. 86). Retrieved from http:// www.endvawnow.org/en/articles/330-what-is-monitoring-and-evaluation-.html?next=331 